\title{
Fração Exalada de Óxido Nítrico no Controlo e Abordagem Terapêutica da Asma
}

\author{
Fractional Exhaled Nitric Oxide in Monitoring and Therapeutic Management of \\ Asthma
}

\author{
Bruno MELO ${ }^{1}$, Patrício COSTA ${ }^{2}$, Ariana AFONSO ${ }^{3}$, Vânia MACHADO ${ }^{3}$, Carla MOREIRA ${ }^{3}$, Augusta GONÇALVES ${ }^{3}$, \\ Jean-Pierre GONÇALVES 2,3 \\ Acta Med Port 2014 Jan-Feb;27(1):59-66
}

\section{RESUMO}

Introdução: A asma é uma doença respiratória crónica caracterizada pela hiper-reactividade e inflamação brônquica. A inflamação brônquica destes doentes pode ser monitorizada através da medição da fração exalada de óxido nítrico. Este estudo tem por objetivo determinar a associação do valor da fração exalada de óxido nítrico com o débito expiratório máximo instantâneo e com o controlo da asma determinado pela Classificação da Iniciativa Global para a Asma.

Material e Métodos: Estudo observacional, analítico e transversal de crianças com asma, 6-12 anos, seguidas na Consulta Externa de Patologia Respiratória do Hospital de Braga. Informação sociodemográfica e clínica colhida através de um questionário. Determinado o valor da fração exalada de óxido nítrico, através do analisador portátil Niox Mino ${ }^{\circledR}$, e do débito expiratório máximo instantâneo, através do debitómetro.

Resultados: A amostra é constituída por 101 crianças asmáticas, 63 (62,4\%) do sexo masculino e 38 (37,6\%) do sexo feminino. A idade média dos participantes na amostra é de $9,18(1,99)$ anos. A regressão logística, realizada com o valor de cutoff obtido pela curva de ROC, revelou que a fração exalada de óxido nítrico tem um efeito estatisticamente significativo $\left(b_{\text {Niveis do } F E N O}=0,85 ; \chi_{\text {Wald }}^{2}(1)=8,71\right.$; $\mathrm{OR}=2,33 ; p=0,003$ ) sobre a probabilidade de mudar de nível de controlo da asma. Por cada nível de fração exalada de óxido nítrico incrementado o odds de passar a não controlada é 2,33 vezes superior.

Discussão: A probabilidade de uma criança asmática mudar o seu nível de controlo da asma, de 'controlada' para 'parcialmente controlada/não controlada', tendo em consideração uma alteração no seu nível da fração exalada de óxido nítrico, aumenta $133 \%$.

Palavras-chave: Criança; Asma; Expiração; Óxido Nítrico; Portugal.

\section{ABSTRACT}

Introduction: Asthma is a chronic respiratory disease characterized by hyper-responsiveness and bronchial inflammation. The bronchial inflammation in these patients can be monitored by measuring the fractional exhaled nitric oxide. This study aims to determine fractional exhaled nitric oxide association with peak expiratory flow and with asthma control inferred by the Global Initiative for Asthma. Material and Methods: Observational, analytical and cross-sectional study of children with asthma, 6-12 years-old, followed in the Outpatient Respiratory Pathology of Braga Hospital. Sociodemographic and clinical information were collected through a questionnaire. fractional exhaled nitric oxide and peak expiratory flow were determined by portable analyzer Niox Mino ${ }^{\circledR}$ and flow meter, respectively. Results: The sample is constituted by 101 asthmatic children, $63(62.4 \%)$ of males and $38(37.6 \%)$ females. The mean age of participants in the sample is 9.18 (1.99) years. The logistic regression performed with the cutoff value obtained by ROC curve, revealed that fractional exhaled nitric oxide $\left(b_{F E N O \text { classes }}=0.85 ; \chi_{\text {wald }}^{2}(1)=8.71\right.$; OR $\left.=2.33 ; p=0.003\right)$ has a statistical significant effect on the probability of changing level of asthma control. The odds ratio of going from "controlled" to "partly controlled/uncontrolled" is 2.33 per each level of fractional exhaled nitric oxide.

Discussion: The probability of an asthmatic children change their level of asthma control, from 'controlled' to 'partly controlled/uncontrolled', taking into account a change in their fractional exhaled nitric oxide level, increases $133 \%$.

Keywords: Child; Asthma; Exhalation; Nitric Oxide; Portugal.

\section{INTRODUÇÃo}

A asma é uma doença respiratória crónica caracterizada pela hiper-reactividade e inflamação brônquica..$^{1,2}$ Os principais sinais e sintomas da asma são a dispneia/dificuldade respiratória, a pieira, o aperto no peito e a tosse. ${ }^{3} \mathrm{O}$ processo fisiopatológico da inflamação é caracterizado pelo envolvimento de várias células e mediadores inflamatórios, salientando os eosinófilos, macrófagos, citoquinas, leucotrienos cisteínicos e óxido nítrico. ${ }^{3-5} \mathrm{~A}$ inflamação crónica das vias aéreas está associada a uma hiper-reactividade brônquica para vários estímulos, a salientar os ácaros, pólens, fumo de tabaco e poluição do $\mathrm{ar}^{3}{ }^{3}$ que conduzem a uma obstrução generalizada das vias aéreas pulmonares e, por consequência, a uma redução do fluxo do ar.

Estima-se que a asma afete cerca de 300 milhões de pessoas em todo o mundo. ${ }^{6,7}$ Esta é a doença crónica mais comum entre as crianças. ${ }^{8}$ De acordo com o Inquérito $\mathrm{Na}-$ cional de Saúde 2005/2006, a prevalência da asma pediátrica, em Portugal, nas crianças com idade inferior a 15 anos, é de $4,94 \%{ }^{9}$

A asma é uma doença com impacto ao nível pessoal, familiar e socioeconómico. ${ }^{10} \mathrm{O}$ seu não controlo conduz à perda de dias escolares, de qualidade de vida e a um aumento do custo no seu tratamento em relação à asma controlada. ${ }^{3,10}$

\footnotetext{
1. Escola de Ciências da Saúde. Universidade do Minho. Braga. Portugal.

2. Saúde Comunitária. Escola de Ciências da Saúde. Universidade do Minho. Braga. Portugal.

3. Serviço de Pediatria. Hospital de Braga. Braga. Portugal.

Recebido: 24 de Março de 2013 - Aceite: 16 de Agosto de 2013 | Copyright @ Ordem dos Médicos 2014
} 
O óxido nítrico foi detetado no ar expirado, pela primeira vez, em $1991^{11}$ e é produzido pelo epitélio brônquico. ${ }^{12} \mathrm{~A}$ Fração Exalada de Óxido Nítrico (FENO) tem vindo a ser estudada ao longo dos últimos anos devido à importância de uma monitorização e uma avaliação precisa do processo inflamatório, uma vez que tanto os sinais e sintomas da asma como a diminuição da função respiratória não são específicos e não refletem necessariamente o processo inflamatório subjacente. ${ }^{13}$ Além disso, a inflamação das vias aéreas está presente independentemente da presença ou ausência de sintomas, do fenótipo da doença ou de ser uma asma ativa ou em remissão, ${ }^{10,14-16}$ contrastando com a obstrução brônquica que é reversível espontaneamente ou após tratamento. ${ }^{3}$ Atendendo a este facto, torna-se, assim, essencial investigar técnicas que permitam monitorizar o estado inflamatório das vias aéreas de forma a permitir um melhor seguimento e deteção precoce das agudizações ou do mau controlo da asma.

As medições do FENO podem ser afetadas por alguns fatores, como a idade, sexo, atopia, fumo do cigarro, comida rica em nitratos, infeções das vias respiratórias, glucocorticóides orais/inalados e antagonistas dos leucotrienos. ${ }^{11,16-22}$

Um dos métodos que permite avaliar a inflamação das vias aéreas é a biópsia brônquica, contudo, este exame é caro e invasivo, não podendo ser repetido rotineiramente. ${ }^{16,23}$ Outro método é a expetoração induzida, no entanto muitas crianças não conseguem expelir as secreções e a obtenção dos resultados é demorada. ${ }^{16}$ Contrariamente a estes dois métodos, a medição do FENO é simples, não invasiva e obtêm-se os resultados rapidamente. ${ }^{24}$ Vários estudos revelaram uma correlação positiva entre o FENO e biópsia brônquica ${ }^{15,25}$ e a análise de expetoração. ${ }^{26-28}$

A American Thoracic Society (ATS) concluiu em 2011 que o FENO tem um papel importante na deteção da inflamação eosinófila das vias aéreas, na determinação da resposta aos corticóides, na monitorização da inflamação das vias aéreas para determinar a necessidade de utilização de corticóides e para verificar a adesão à terapêutica com corticóides. ${ }^{29}$

O aumento do FENO, em crianças, tem sido apontado como preditor de recaída da asma após a retirada dos corticóides. ${ }^{30,31}$

Em 2009, a ATS identificou como questões para futuras investigações a utilidade da medição do FENO como ferramenta para monitorizar o controlo da asma e a clarificação da relação entre o FENO e outros parâmetros de controlo da asma. ${ }^{32}$ Neste sentido, surgiu a ideia e a oportunidade de realizar este trabalho.

O presente estudo tem como objetivo avaliar a associação entre o valor do FENO com o débito expiratório máximo instantâneo (DEMI) e com o controlo da asma determinado pela Classificação GINA (Iniciativa Global para a Asma).

\section{MATERIAL E MÉTODOS}

Estudo observacional, analítico e transversal realizado no Serviço de Pediatria do Hospital de Braga (HB). A população em estudo foi constituída por crianças asmáticas com idade entre os seis e doze anos seguidas na Consulta Externa de Patologia Respiratória (CEPR) do HB. Foi utilizada uma amostra de conveniência constituída por 101 crianças com asma que compareceram na CEPR do HB.

Os critérios de inclusão do estudo foram: apresentar diagnóstico de asma; idade compreendida entre os seis e doze anos, inclusive, e frequentar a CEPR do HB.

Os critérios de exclusão foram: não apresentar diagnóstico de asma; idade menor que seis anos ou maior que doze anos; não frequentar a CEPR do HB e recusa em colaborar no estudo (Fig. 1).

Para a obtenção dos dados foi elaborado um questionário confidencial, anónimo e a ser preenchido pelo investigador, que incluía as características demográficas das crianças asmáticas e dos pais, a terapêutica e o controlo da asma. O nível de controlo da asma (controlada,

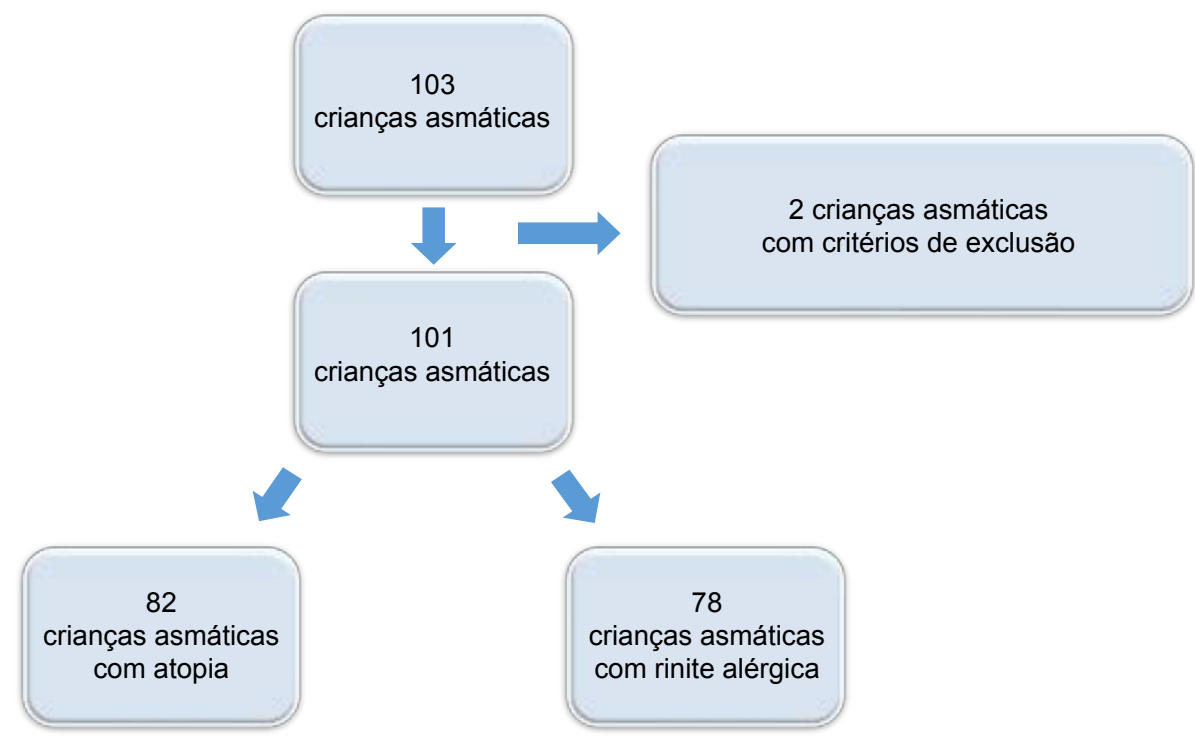

Figura 1 - Diagrama das crianças asmáticas incluídas no estudo 
parcialmente controlada e não controlada) foi obtido a partir da resposta a cinco perguntas realizadas com base na GINA. ${ }^{3}$

O questionário, antes de ser aplicado, foi sujeito a um pré-teste, sendo aplicado a dez crianças asmáticas.

Antes do preenchimento do questionário, foi obtido o consentimento informado dos responsáveis legais das crianças asmáticas.

A determinação instantânea do valor do FENO era realizada através do analisador portátil eletroquímico Niox Mino ${ }^{\circledR}$ (Aerocrine, Suécia), ${ }^{33-36}$ seguindo as recomendações da ATS e da European Respiratory Society (ERS). ${ }^{37}$ Foi realizada uma única medição do FENO em cada criança asmática $^{38,39}$ e o fluxo expiratório exalado tinha uma duração de seis segundos..$^{40,41}$

Após determinação do valor do FENO, era encontrado o valor do débito expiratório máximo instantâneo (DEMI) através do debitómetro segundo as orientações da ATS/ ERS. ${ }^{42} \mathrm{~A}$ medição do DEMI era repetida três vezes, ficando-se com o valor mais elevado das três medições. ${ }^{43}$ Este valor era comparado com valores normais de referência pediátricos. ${ }^{44}$

O peso, altura e perímetro abdominal foram determinados segundo o Guia de Avaliação do Estado Nutricional

Tabela 1 - Análise descritiva relativa à terapêutica e controlo da asma

\begin{tabular}{|c|c|c|}
\hline & n (\%) & Média (DP) \\
\hline \multicolumn{3}{|l|}{ Classes do FENO*: } \\
\hline - Baixo & $0(0)$ & \\
\hline - Normal & $36(35,6)$ & \\
\hline - Intermédio & $17(16,8)$ & \\
\hline - Elevado & $48(47,5)$ & \\
\hline
\end{tabular}

Valor do FENO (ppb)

$38,19(27,74)$

Valor do DEMI ${ }^{\star \star}(\mathrm{L} / \mathrm{min})$

$194,36(61,06)$

Terapêutica efetuada:

- $\quad$ Agonista $\beta 2$ de ação rápida

- Corticosteróides inalados $(\mathrm{Cl})$

- $\quad$ Antileucotrienos (AntL)

$23(22,8)$

- $\quad$ Agonistas $\beta 2$ de ação longa

- Teofilina

- Glucocorticóide oral

- Imunoterapia

- Não toma medicação

- $\quad \mathrm{Cl}+$ Agonistas $\beta 2$ de ação longa

- $\quad \mathrm{Cl}+$ Agonistas $\beta 2$ de ação longa + AntL

- $\mathrm{Cl}+$ AntL

Infeção respiratória recente (últimas 4 semanas)

$\operatorname{Sim}$

$15(14,9)$

Nas últimas 4 semanas:

- Sintomas diurnos mais de $2 x /$ semana

Sim

- Sintomas/despertares noturnos

Sim

$12(11,9)$

- $\quad$ Atividades ou exercícios limitados pela asma

$\operatorname{Sim}$

- Necessidade de medicação de alívio mais de 2x/semana

$\operatorname{Sim}$

- $\quad<80 \%$ do valor previsto do DEMI

$\operatorname{Sim}$

Classificação GINA ${ }^{\star \star *}$ :

- Controlada

- Parcialmente controlada

- Não controlada

$14(13,9)$

* Fração exalada de óxido nítrico; ** Débito expiratório máximo instantâneo; *** Iniciativa Global para a Asma 
Infantil e Juvenil. ${ }^{45}$

O estudo foi aprovado pelas Comissões Executiva e Ética do Hospital de Braga.

Os dados recolhidos foram introduzidos e analisados com recurso ao IBM SPSS Statistical v.20. A normalidade das variáveis contínuas foi analisada através dos testes de Kolmogorov-Smirnov e Shapiro-Wilk, ${ }^{46}$ tendo-se optado pela utilização de testes não-paramétrico para as variáveis FENO e DEMI. A caraterização da amostra foi efetuada com recurso à estatística descritiva (distribuição de frequências, medidas de tendência central e medidas de dispersão). A associação entre as variáveis qualitativas ordinais foi analisada com o coeficiente de correlação ordinal de Spearman. Para avaliar a significância do nível do FENO, atopia, rinite alérgica e infeção respiratória recente (IRC) sobre a probabilidade de ter um nível de controlo 'controlada' ou 'parcialmente controlada/não controlada', segundo a GINA, recorreu-se à regressão logística e à construção de uma curva ROC (receiver operating characteristic).

A variável IRC foi definida como infeção respiratória nas últimas quatro semanas.

As perguntas relativas ao nível de controlo da asma foram definidas como: presença de sintomas durante o dia, mais de duas vezes por semana, sugestivos de asma, nas últimas quatro semanas; presença de sintomas noturnos durante a noite, sugestivos de asma, nas últimas quatro semanas; presença de restrições na realização das suas atividades nas últimas quatro semanas; toma de medicação, para além da tomada, diariamente, de forma crónica, mais de duas vezes por semana, nas últimas quatro semanas; valor do DEMI medido com o debitómetro $<80 \%$ do valor previsto.

A variável FENO foi classificada como: FENO baixo - < 5 ppb (parte por bilião), FENO normal $-<21$ ppb, FENO intermédio - entre 21-35 ppb e FENO alto - > 35 ppb. ${ }^{32,39}$

A variável Classificação GINA, que avalia o nível de controlo da asma de acordo com a GINA, foi dividida em três níveis: controlada, parcialmente controlada e não controlada.
Foi considerado como nível de significância estatística o valor de $p<0,05$.

\section{RESULTADOS}

Dos 103 casos elegíveis, em apenas dois houve recusa de participação no estudo. A amostra é constituída por 101 crianças asmáticas, $63(62,4 \%)$ do sexo masculino e $38(37,6 \%)$ do sexo feminino. A idade média (DP) dos participantes na amostra é de 9,18 $(1,99)$ anos. A maioria dos participantes apresentava atopia $(n=82 ; 81,2 \%)$, rinite alérgica $(n=78 ; 77,2 \%)$, um percentil $(P)$ de perímetro abdominal $<75(n=59 ; 58,4 \%)$ e vivia em meio rural $(n=56$; $55,4 \%)$.

O valor médio do FENO na amostra é de $38,19(27,74)$ ppb e a maioria dos indivíduos encontra-se distribuído pelas classes normal $(n=26 ; 35,6 \%$ ) e elevado ( $n=48$; $47,5 \%$ ) do FENO. Relativamente à terapêutica, a maioria dos indivíduos não toma medicação $(n=34 ; 33,7 \%)$. Em relação ao controlo da asma, os resultados revelaram que a maioria dos indivíduos se encontrava parcialmente controlada, $(n=59 ; 58,4 \%$ ) (Tabela 1$)$.

Os dados revelam que a maioria dos pais não fuma e não tem história familiar de atopia. Contudo, entre os pais, verifica-se que a percentagem das mães que fumam é menor ( $n=10 ; 9,9 \%)$ e a percentagem de mães com história familiar de atopia é maior ( $n=33 ; 32,7 \%$ ). O número de mães a apresentar história familiar de asma é superior $(n=$ $54 ; 53,5 \%)$ à dos pais $(n=46 ; 45,5 \%)$.

No presente estudo verificou-se a inexistência de correlação entre os valores do FENO e os valores do DEMI, $r_{s}=0,154, p=0,125$. Pelo contrário, há uma correlação significativa, positiva, de magnitude moderada, segundo a classificação de Cohen ${ }^{48}$ entre a Classificação GINA e as classes do FENO, $r_{s}=0,405, p<0,001$.

Relativamente à terapêutica, as crianças asmáticas, que apenas estão medicadas com corticosteróides inalados $(\mathrm{Cl})$, revelam ter um menor valor do FENO em comparação com as crianças que tomam Antileucotrienos (AntL), $\mathrm{Cl}+$ AntL ou que não tomam nenhuma medicação (Fig. 2).

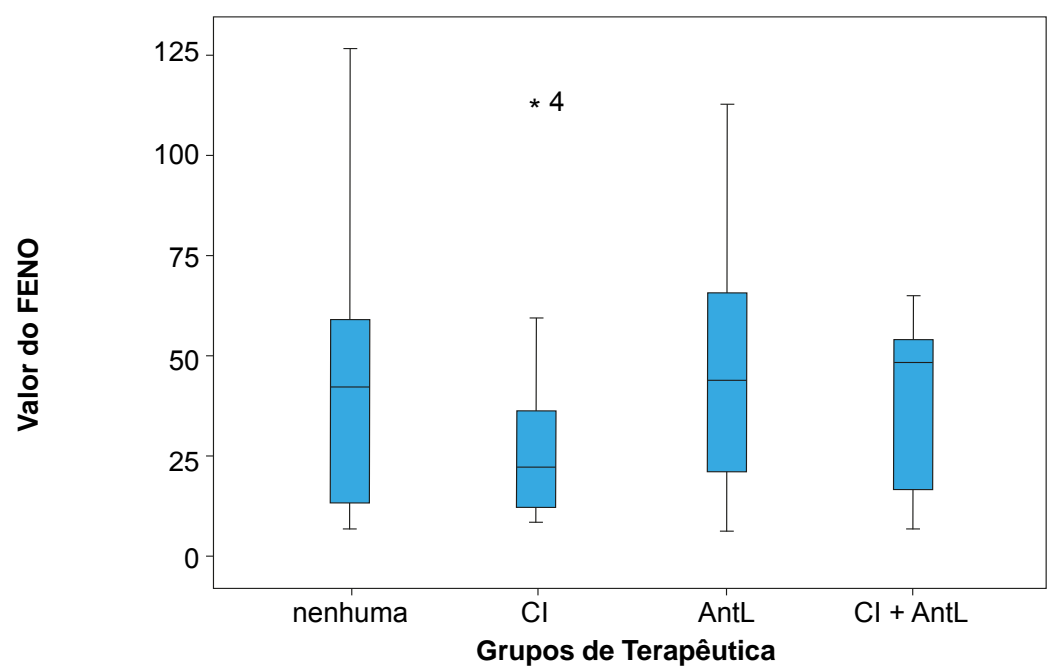

Figura 2 - Valores do FENO segundo os grupos de terapêutica, onde as crianças asmáticas estão inseridas 
Tabela 2 - Coeficientes do modelo de regressão logística da variável Classificação GINA* em função das classes do FENO**, IRC***, atopia e rinite alérgica

\begin{tabular}{|c|c|c|c|c|c|c|}
\hline & $B$ & S.E. & $\chi_{\text {Wald }}^{2}$ & Valor de $p$ & OR & I.C. a $95 \%$ \\
\hline Classes do Feno & 0,85 & 0,29 & 8,71 & 0,003 & 2,33 & ]1,33; 4,10[ \\
\hline IRC & $-0,94$ & 0,83 & 1,25 & 0,263 & 0,39 & ]0,08; 2,02[ \\
\hline Atopia & 0,26 & 0,73 & 0,13 & 0,723 & 1,29 & ]0,31; 5,35[ \\
\hline Rinite alérgica & 1,07 & 0,78 & 1,89 & 0,169 & 2,92 & ]0,63; 13,40 \\
\hline Constante & $-0,98$ & 1,21 & 0,65 & 0,420 & 0,38 & - \\
\hline
\end{tabular}

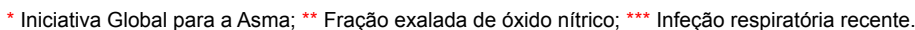

Tabela 3 - Classificação observada e prevista das crianças asmáticas (cutoff é 0,739)

Classificação GINA*

Controlada Parcialmente controlada / não controlada

$\begin{array}{llccc}\begin{array}{c}\text { Classificação } \\ \text { GINA }\end{array} & \text { Controlada } & 18 & 10 & 64,3 \\ & \text { Parcialmente controlada / não controlada } & 22 & 51 & 69,9 \\ & & & 68,3 \\ & \text { Percentagem Global } & & \\ \end{array}$

* Iniciativa Global para a Asma

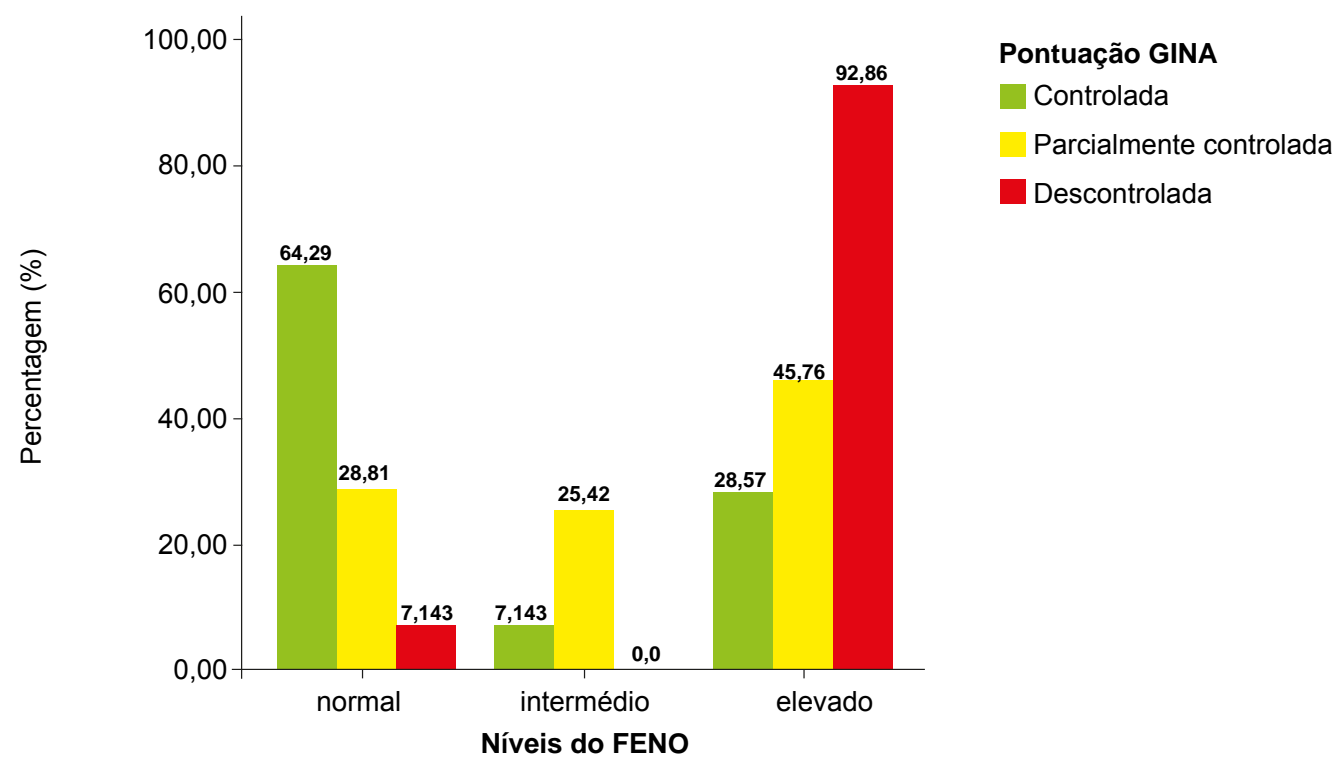

Figura 3 - Percentagem de crianças asmáticas de acordo com os níveis de controlo de asma da GINA e classes do FENO

A Fig. 3 representa a percentagem de pacientes em cada grupo, de acordo com as classes do FENO e a Classificação GINA. A maioria dos doentes com asma controlada e não controlada, segunda a GINA, estão corretamente classificados pelas classes do FENO (64,29\% e 92,86\%, respetivamente). No entanto, os doentes com asma parcialmente controlada estão distribuídos maioritariamente pelas classes do FENO elevado e normal $(45,76 \%$ e $28,81 \%$, respetivamente).

A regressão logística, realizada com o valor de cutoff obtido pela curva de ROC com o objetivo de maximizar a sensibilidade e especificidade, revelou que a IRC $\left(b_{I R C}=-, 94 ; \chi^{2}{ }_{\text {Wald }}(1)=1,25 ; p=0,263\right)$, a atopia $\left(b_{\text {Atopia }}=0,26\right.$; $\left.\chi_{\text {Wald }}^{2}(1)=0,13 ; p=0,723\right)$ e a rinite alérgica $\left(b_{\text {Rinite Alérgica }}=\right.$ 
1,$\left.07 ; \chi_{\text {wald }}^{2}(1)=1,89 ; p=0,169\right)$ não apresentam um efeito estatisticamente significativo sobre a probabilidade de mudar de nível de controlo da asma. Pelo contrário, a variável classes do FENO $\left(b_{\text {Classes do FENO }}=0,85 ; \chi_{\text {wald }}^{2}(1)=8,71\right.$; $\mathrm{OR}=2,33 ; p=0,003)$ tem um efeito estatisticamente significativo (Tabela 2). O rácio das chances de passar de 'controlada' para 'parcialmente controlada/não controlada' é de 2,33 por cada classe do FENO, ou seja, quando uma criança passa de uma determinada classe do FENO para outra, as chances de passar de 'controlada' para 'parcialmente controlada/não controlada' aumentam $133 \%$, segundo a expressão \%Rácio Chances $=100 \times\left[\exp \left(\beta_{j}\right)-1\right] .{ }^{46}$ Este modelo de regressão logística é significativo $[\mathrm{G} 2(\mathrm{df})=15,799$, $p<0,001$; Nagelkerke R2 $=0,209]$. Procedeu-se também à classificação dos sujeitos amostrados, observando-se uma percentagem de classificação correta de 68,3\% (Tabela 3). Este valor é consideravelmente superior à percentagem proporcional de classificações corretas por acaso (59,9\%). É apresentada uma sensibilidade de $69,9 \%$ e uma especificidade de $64,3 \%$ (Tabela 3 ), bem como uma capacidade discriminante aceitável (ROC $c=0,745 ; p<0,001)$, segundo Hosmer e Lemeshow. ${ }^{49}$

\section{DISCUSSÃO}

O objetivo do tratamento da asma é o de atingir e manter o controlo das manifestações clínicas da doença por períodos prolongados, ${ }^{3}$ contudo, alguns estudos revelaram que este objetivo ainda está longe de ser alcançado. ${ }^{50,51} \mathrm{~A}$ GINA, com o objetivo de auxiliar os médicos no controlo da asma, estabeleceu uma classificação do controlo da asma dividida em três níveis: controlada, parcialmente controlada e não controlada. No entanto, esta classificação não incorpora uma avaliação objetiva da inflamação das vias aéreas. Assim, é essencial investir e explorar novas técnicas, que permitam aprofundar o conhecimento sobre o controlo da asma de uma forma simples e rápida, sendo o FENO, de acordo com recente literatura, um desses exemplos.

No presente estudo, foram avaliadas crianças asmáticas com idade compreendida entre os seis e os doze anos, inclusive. A partir dos seis anos porque, embora os sintomas de asma sejam comuns em idades pré-escolares, estes são frequentemente transitórios, ${ }^{52}$ e também porque a GINA diferencia o diagnóstico de asma acima dos seis anos. O limite superior dos doze anos foi definido por razões operacionais e de contenção de custos.

No atual estudo, o número de rapazes que apresenta diagnóstico de asma é superior ao número de raparigas, o que vai de encontro ao reportado em vários estudos. ${ }^{3,52-55} \mathrm{~A}$ maioria das crianças, $58,4 \%$, não apresenta um risco aumentado de obesidade abdominal $(p<75)$ e 19,8\% têm obesidade abdominal $(p>90)$. Ao contrário do dito por Bacharier et $a l,{ }^{52}$ aqui a prevalência da asma não é superior nas crianças com história familiar de atopia.

O presente estudo revela que não há correlação entre os valores do FENO e os valores do DEMI, estando de acordo com o estudo de Pijnenburg et al ${ }^{56}$ Este resultado pode ser explicado em função da avaliação de diferentes características do controlo da asma por parte do FENO, que avalia a inflamação das vias aéreas, e do DEMI, que avalia a função pulmonar. Deste modo, os dois exames complementam-se na avaliação do controlo da asma.

Este estudo demonstra uma correlação positiva, de magnitude moderada ${ }^{48}$ e estatisticamente significativa entre os níveis de controlo da asma estabelecidos pela GINA e as classes do FENO.

Um dos resultados obtidos por este trabalho foi a constatação de que as crianças asmáticas, que tomam diariamente corticosteróides, apresentam, na sua maioria, valores do FENO inferiores aos das crianças que tomam outro tipo de medicação. Estes resultados são consonantes com o que foi referido em The European Respiratory Monograph por Alving e Malinovschi. ${ }^{57}$

Relativamente à Fig. 3, uma possível justificação para algumas crianças classificadas pela GINA como parcialmente controlada ou não controlada e que, com base na classificação do FENO, se encontram no nível normal, será devido ao facto do FENO não apresentar uma boa correlação com a inflamação mediada por neutrófilos. ${ }^{10,58}$ É de realçar que os valores do FENO podem ser significativamente afetados por alguns fatores que não foram excluídos no atual estudo, como a medicação com corticosteróides inalados, rinite alérgica, infeção respiratória recente e alimentação, o que pode explicar, em parte, o facto de algumas crianças classificadas pela GINA como controladas ou parcialmente controladas estarem, comparativamente às classes do FENO, em patamares superiores.

No presente estudo, não se obtiveram valores absolutos do FENO para predizer a perda de controlo da asma, mas sim a probabilidade da criança asmática deixar de estar controlada para estar parcialmente controlada/não controlada, segundo os critérios da GINA. De acordo com a regressão logística, realizada com o valor de cutoff obtido pela curva de ROC, a mudança do nível de controlo da asma não é afetada significativamente pela IRC, atopia ou rinite alérgica. No entanto, a variável classes do FENO têm um efeito estatisticamente significativo sobre a probabilidade da criança asmática mudar o seu nível de controlo da asma. Deste modo, quando uma criança asmática muda de nível do FENO, a oportunidade desta passar do nível 'controlada' para o nível 'parcialmente controlada/não controlada' aumentam $133 \%$.

Obteve-se uma percentagem de $68,3 \%$ relativamente à classificação correta das crianças asmáticas, ou seja, $68,3 \%$ das crianças são corretamente classificadas pelos três níveis do FENO. Este valor é superior em 59,9\% à percentagem proporcional de classificações corretas por acaso, demonstrando a utilidade do modelo para classificar novas observações. É apresentada uma capacidade discriminante aceitável, ${ }^{49}$ pela curva de ROC, bem como uma sensibilidade $(69,9 \%)$ e especificidade $(64,3 \%)$.

Algumas limitações poderão ser apontadas a este estudo nomeadamente a amostra ser de conveniência; a não exclusão das crianças asmáticas com características que poderiam afetar as medições do FENO, no entanto, a sua 
não exclusão permite manter as conjunturas habituais da prática clínica e obter resultados que podem ser extrapolados para a população geral de crianças asmáticas e não só para o grupo de crianças asmáticas que não tivessem essas características ${ }^{17}$; a variabilidade diurna do $\mathrm{DEMI}^{59}$ e a possível má execução da técnica, na realização do exame, por parte da criança. Contudo, na ausência de instrumento de avaliação do controlo asma validado para população pediátrica em Portugal, decidiu-se avaliar os doentes segundo as linhas de orientação da GINA, que incluíam a medição do DEMI através do debitómetro.

\section{CONCLUSÃO}

Este estudo demonstrou que a maioria das crianças asmáticas, da amostra estudada, tem a sua doença controlada ou parcialmente controlada.

\section{REFERÊNCIAS}

1. Gelb A, Taylor C, Simmons M, Shinar C, Gutierrez C, Zamel N. Role of add-on zileuton on total exhaled, large airway, and small airway/alveolar nitric oxide in moderate-severe persistent adult asthmatics on fluticasone $250 \mu \mathrm{g} /$ Salmeterol $50 \mu \mathrm{g}$. Pulm Pharmacol Ther. 2009;22:516-21.

2. Ozier A, Girodet PO, Bara I, Tunon de Lara JM, Marthan R, Berger P. Control maintenance can be predicted by exhaled NO monitoring in asthmatic patients. Respiratory Med. 2011;105:989-96.

3. The Global Strategy for Asthma Management and Prevention, Global Initiative for Asthma (GINA), 2011. [Consultado 2012 Abr 20]. Disponível em: http://www.ginasthma.org/.

4. Busse W, Lemanske R. Asthma. N Engl J Med. 2001;344:350-62.

5. Longo D, Kasper D, Jameson J, Fauci A, Hauser S, Loscalzo J, editors. Harrison`s-Principles of Internal Medicine. 18th ed. Philadelphia: McGraw-Hill; 2011.

6. Masoli M, Fabian D, Holt S, Beasley R. The global burden of asthma: executive summary of the GINA Dissemination Committee report. Allergy. 2004;59:469-78.

7. Masoli M, Fabian D, Holt S, Beasley R. Global Burden of Asthma, Global Initiative for Asthma (GINA), 2004. [Consultado 2012 Abr 28]. Disponível em: http://www.ginasthma.org/reports-global-burden-of-asthma.html.

8. World Health Organization. Asthma - Fact sheet. [Consultado 2012 Abril 28]. Disponível em: http://www.who.int/mediacentre/factsheets/fs $307 /$ en/index.html.

9. Instituto Nacional de Estatística. Inquérito Nacional de Saúde 2005/2006. Lisboa: INE; 2009.

10. Estepa R, Crespo M, Moreno S, Cáceres J, Muñoz P, Calvo C. Revisión sistemática de la utilidad de la determinación del óxido nítrico espirado en el control del asma infanto-juvenil. Sevilla: Agencia de Evaluación de Tecnologías Sanitarias de Andalucía; 2011.

11. Lopes C, Fonseca J, Silva J, Castel-Branco M. Óxido nítrico exalado: utilização clínica na Asma. Rev Port Imunoalergol. 2005;13:7-17.

12. Frank TL, Adisesh A, Pickering AC, Morrison JF, Wright T, Francis H, et al. Relationship between exhaled nitric oxide and childhood asthma. American J Respir Crit Care Med. 1998;158:1032-6.

13. Rosi E, Ronchi MC, Grazzini M, Duranti R, Scano G. Sputum analysis, bronchial hyperresponsiveness, and airway function in asthma: results of a factor analysis. J Allergy Clin Immunol. 1999;103:232-7.

14. National Institutes of Health. National Asthma Education and Prevention Program. Expert panel report: Guidelines for the diagnosis and management of asthma, 2002. [consultado 2012 Abril 26]. Disponível em: http:// www.nhlbi.nih.gov/guidelines/asthma/asthmafullrpt.pdF.

15. Van den Toorn LM, Overbeek SE, Jongste JC, Leman K, Hoogsteden $\mathrm{HC}$, Prins JB. Airway inflammation is present during clinical remission of atopic asthma. Am J Respir Crit Care Med. 2001;164: 2107-13.

16. Sandrini A, Taylor D, Thomas $P$, Yates D. Fractional exhaled nitric oxide in asthma: an update. Respirology. 2010;15: 57-70.

17. Alvarez-Gutiérrez FJ, Medina-Gallardo JF, Pérez-Navarro $P$, Martín-Villasclaras JJ, Martin Etchegoren B, Romero-Romero B, et al. Relationship of the Asthma Control Test (ACT) with lung function, levels of exhaled nitric oxide and control according to the Global Initiative for Asthma (GINA). Arch Bronconeumol. 2010;46:370-7.
Entre os níveis de controlo da asma estabelecidos pela GINA e os níveis do FENO há uma correlação positiva. No entanto, entre os valores do FENO e os valores do DEMI não há correlação.

A probabilidade de uma criança asmática mudar o seu nível de controlo da asma, de 'controlada' para 'parcialmente controlada/não controlada', tendo em consideração uma alteração no seu nível do FENO, aumenta $133 \%$.

\section{CONFLITOS DE INTERESSE}

Os autores declaram a inexistência de conflitos de interesse.

\section{FONTES DE FINANCIAMENTO}

Os autores declaram a inexistência de financiamento.

18. Hervás D, Milán J, Garde J. Differences in exhaled nitric oxide in atopic children. Allergol Immunopathol. 2008;36:331-5.

19. Buchvald F, Baraldi E, Carraro S, Gaston B, De Jongste J, Pijnenburg $\mathrm{MW}$, et al. Measurements of exhaled nitric oxide in healthy subjects age 4 to 17 years. J Allergy Clin Immunol. 2005;115:1130-6.

20. Tsang KW, Ip SK, Leung R, Tipoe GL, Chan SL, Shum IH, et al. Exhaled nitric oxide: the effects of age, gender and body size. Lung 2001;179:83-91.

21. Bisgaard $\mathrm{H}$, Loland $\mathrm{L}$, Anhol $\mathrm{J}$. NO in exhaled air of asthmatic children is reduced by the Leukotriene Receptor Antagonist Montelukast. Am J Respir Crit Care Med. 1999;160:1227-31.

22. Gelb A, Taylor C, Shinar C, Gutierrez C, Zamel N. Effect of fluticasone $250 \mu \mathrm{g} /$ Salmeterol $50 \mu \mathrm{g}$ and montelukast on exhaled nitric oxide in asthmatic patients. Can Respir J. 2008;15:193-8.

23. Fritsch M, Uxa S, Horak F Jr, Putschoegl B, Dehlink E, Szepfalusi Z, et al. Exhaled nitric oxide in the management of childhood asthma: A prospective 6-months study. Pediatr Pulmonol. 2006;41:855-62.

24. Deykin A. Targeting biologic markers in asthma - is exhaled nitric oxide the bull's eyes? N Engl J Med. 2005;352:2233-5.

25. Payne DN, Adcock IM, Wilson NM, Oates T, Scallan M, Bush A. Relationship between exhaled nitric oxide and mucosal eosinophilic inflammation in children with difficult asthma, after treatment with oral prednisolone. Am J Respir Crit Care Med. 2001;164:1376-81.

26. Smith AD, Cowan JO, Filsell S, McLachlan C, Monti-Sheehan G, Jackson $\mathrm{P}$, et al. Diagnosing asthma - Comparisons between exhaled nitric oxide measurements and conventional tests. Am J Respir Crit Care Med. 2004:169:473-8.

27. Berlyne GS, Parameswaran K, Kamada D, Efthimiadis A, Hargreave FE. A comparison of exhaled nitric oxide and induced sputum as markers of airway inflammation. J Allergy Clin Immunol. 2000;106:638-44.

28. Piacentini GL, Bodini A, Costella S, Vicentini L, Mazzi P, Sperandio S, et al. Exhaled nitric oxide and sputum eosinophil markers of inflammation in asthmatic children. Eur Respir J. 1999;13:1386-90

29. Dweik RA, Boggs PB, Erzurum SC, Irvin CG, Leigh MW, Lunderg JO, et al. An official ATS clinical practice guideline: Interpretation of Exhaled Nitric Oxide Levels (FENO) for clinical applications. Am J Respir Crit Care Med. 2011;184:602-15

30. Pijnenburg M, Hofhuis W, Hop W, De Jongste J. Exhaled nitric oxide predicts asthma relapse in children with clinical asthma remission. Thorax. 2005;60:215-8

31. Zacharasiewicz A, Wilson N, Lex C, Erin EM, Li AM, Hansel T, et al. Clinical use of noninvasive measurements of airway inflammation in steroid reduction in children. Am J Respir Crit Care Med. 2005;171:1077-82.

32. Reddel H, Taylor D, Bateman E, Boulet L, Boushey H, Busse W, et al. An official American Thoracic Society/European Respiratory Society Statement: Asthma control and exacerbations. Am J Respir Crit Care Med. 2009;180:59-99.

33. Gill M, Graff G, Adler A, Dweik R. Validation study of fractional exhaled nitric oxide measurements using a handheld monitoring device. J Asthma. 2006;43:731-4

34. Alving K, Janson C, Nordvall L. Performance of a new hand-held device 
for exhaled nitric oxide measurement in adults and children. Respir Res. 2006;7:67.

35. Menzies D, Nair A, Lipworth B. Portable exhaled nitric oxide measurement: comparison with the "gold standard" technique. Chest. 2007;131:410-4.

36. McGill C, Malik G, Turner S. Validation of a hand-held exhaled nitric oxide analyzer for use in children. Pediatr Pulmonol. 2006;41:1053-7.

37. ATS e ERS. ATS/ERS recommendations for standardized procedures for the online and offline measurement of exhaled lower respiratory nitric oxide and nasal nitric oxide, 2005. Am J Respir Crit Care Med. 2005;171:912-30.

38. Baptist A, Khan F, Wang Y, Ager J. Exhaled nitric oxide measurements in hospitalized children with asthma. J Asthma. 2008;45:670-4.

39. Melhorar o Diagnóstico e o Tratamento da Asma através da Medição da FENO. [consultado 2012 Jun 20]. Disponível em: http://www.teprel.com/ files/catalogos/pneumologia/AEROCRINE\%20FeNO\%20PT.pdf.

40. Prado O, Pérez-Yarza EG, Ruiz A, Murua J, Aranburu J, Knorr J. Fracción exhalada de óxido nítrico: validación de la medida con tiempo de espiración de $6 \mathrm{~s}$ con dos analizadores diferentes. An Pediatr. 2008;69:221-6.

41. Ito $\mathrm{Y}$, Adachi $\mathrm{Y}$, Itazawa T, Okabe $\mathrm{Y}$, Adachi $\mathrm{YS}$, Katsumuma T, et al. Comparison of exhalation time methods ( $6 \mathrm{sec}$ vs. $10 \mathrm{sec}$ ) of a handheld exhaled nitric oxide Analyzer. Pediatr Pulmonol. 2010;45:1005-8.

42. Miller M, Hankinson J, Brusasco V, Burgos F, Casaburi R, Coates A, et al. ATS/ERS Task Force standardisation of lung function testing: Standardisation of spirometry. Eur Respir J. 2005;26:319-38.

43. Direção Geral da Saúde. Manual de Boas Práticas na Asma. Programa Nacional de Controlo da Asma. Lisboa: DGS; 2001.

44. Paediatric Normal Values. [consultado 2012 Maio 20]. Disponível em: http://www.peakflow.com/paediatric_normal_values.pdf.

45. Rito A, Breda J, Carmo I. Instituto Nacional de Saúde Doutor Ricardo Jorge e Direção-Geral da Saúde. Guia de avaliação do estado nutricional infantil e juvenil. Lisboa: INSA; 2010.

46. Marôco J. Análise Estatística com o PASW statistics. Pêro Pinheiro: Report Number; 2010.

47. Martins C. Manual de análise de dados quantitativos com recurso ao $\mathrm{IBM}^{\circledR}$ SPSS ${ }^{\circledR}$ : Saber decidir, fazer, interpretar e redigir. Braga: Psiquilí- brios Edições; 2011

48. Cohen J. Statistical power analysis for the behavioral sciences. 2nd ed New Jersey: Lawrence Erlbaum Associates; 1988.

49. Hosmer D, Lemeshow S. Applied Logistic Regression. 2nd ed. New York: John Wiley \& Sons; 2000.

50. Ferreira J, Silveira P, Marques J. Estudo nacional de qualidade de vida na asma - Aplicação da Asthma Quality of Life Questionnaire de Marks (AQLQ-M) na população portuguesa. Rev Port Pneumol. 2008;14:45983.

51. Dockrell M, Partridge $M$, Valovirta $E$. The limitations of severe asthma: the results of a European survey. Allergy. 2007;62:134-41.

52. Bacharier LB, Boner A, Carlsen KH, Eigenmann PA, Frischer T, Gotz $\mathrm{M}$, et al. Diagnosis and treatment of asthma in childhood: a PRACTALL consensus report. Allergy. 2008;63:5-34.

53. Nordvall SL, Janson C, Kalm-Stephens P, Foucard T, Torén K, Alving K Exhaled nitric oxide in a population-based study of asthma and allergy in schoolchildren. Allergy. 2005;60:469-75.

54. Taylor D, Mandhane P, Greene J, Hancox R, Filsell S, McLachlan C, et al. Factors affecting exhaled nitric oxide measurements: the effect of sex. Respir Res. 2007;8:82.

55. Yavus ST, Civelek E, Sahiner UM, Buyuktiryaki AB, Tuncer A, Karabulut $E$, et al. Identifying uncontrolled asthma in children with the childhood asthma control test or exhaled nitric oxide measurement. Ann Allergy Asthma Immunol. 2012;109:36-40.

56. Pijnenburg MW, Hofhuis W, Hop WC, De Jongste JC. Exhaled nitric oxide predicts asthma relapse in children with clinical asthma remission. Thorax. 2005;60:215-8.

57. Alving K, Malinovschi A. Basic aspects of exhaled nitric oxide. In: Horvath I, Jongste JC, editors. European Respiratory monograph 49: Exhaled Biomarkers. London: ERS; 2010.

58. Costa E, Rizzo J, Machado A, França A, Sano F, Wandalsen G, et al Marcadores inflamatórios no monitoramento do controle da asma: revisão. Rev Bras Alerg Imunopatol. 2011;34:193-202.

59. Troyanov S, Ghezzo H, Cartier A, Malo J. Comparison of circadian variations using FEV1 and peak expiratory flow rates among normal and asthmatic subjects. Thorax. 1994;49:775-80. 


\section{Fração Exalada de Óxido Nítrico no Controlo e Abordagem Terapêutica da Asma

\author{
Acta Med Port 2014:27:59-66
}

Publicado pela Acta Médica Portuguesa, a Revista Científica da Ordem dos Médicos

Av. Almirante Gago Coutinho, 151

1749-084 Lisboa, Portugal.

Tel: +351218428215

E-mail: submissao@actamedicaportuguesa.com

www.actamedicaportuguesa.com

ISSN:0870-399X | e-ISSN: 1646-0758

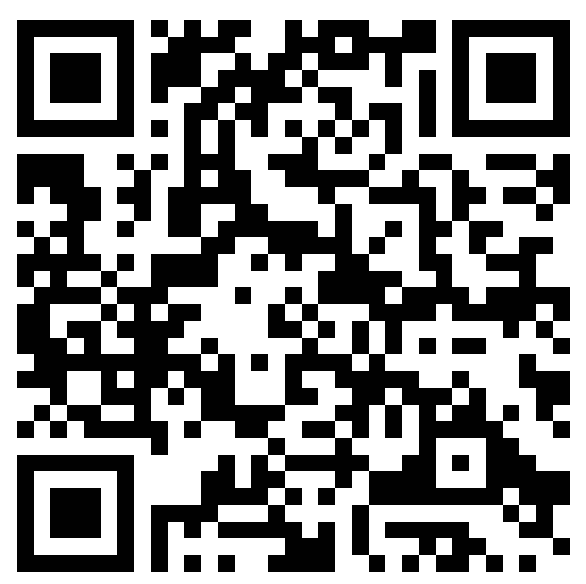

